\title{
Situation Awareness to Support Decision-Making among Emergency Care Practitioners
}

\author{
Harris Shah Abd Hamid \\ HEPSU, Department of Human Sciences \\ Loughborough University \\ Loughborough \\ LE1 1 3TU England \\ H.S.Abd-Hamid@lboro.ac.uk
}

\author{
Patrick Waterson \\ HEPSU, Department of Human Sciences \\ Loughborough University \\ Loughborough \\ LE11 3TU England \\ P.Waterson@1boro.ac.uk
}

\author{
Sue Hignett \\ HEPSU, Department of Human Sciences \\ Loughborough University \\ Loughborough \\ LE11 3TU England \\ S.M.Hignett@lboro.ac.uk
}

\begin{abstract}
Motivation - To understand a new work role in emergency medical services, the relationship between situation awareness (SA) and decision-making among emergency care practitioners (ECPs) is examined. Research approach - A scoping study was completed and involved observations (36 hours of ride-outs and 4 hours of sit-ins) and semi-structured interviews with a group of ECPs and Triage Nurses at an ambulance control room. A subject matter expert was consulted to validate a communication map derived from the observations and interviews. Findings/Design - The results show that system's factors could affect the creation of SA and decision-making. As a result, a framework for SA and decision-making among ECPs was developed. Originality/Value - This study extends the study of SA into emergency medical services domain. It also contributes in understanding a new practitioner role in the UK. Take away message - Factors related to the development of SA should be understood to support decisionmaking among ECPs and thereby improve the quality of care in emergency medicine.
\end{abstract}

\section{Keywords}

situation awareness, emergency care practitioner, system approach

\section{INTRODUCTION}

There seems to be common agreement that human factors and ergonomics can contribute to improve patient safety. The last few years see increased research activity involving human factors and patient safety. This paper highlights situation awareness (SA) and its relationship with decision-making as factors that can contribute to patient safety. In addition, a socio-technical system framework that links SA and decision-making among emergency care practitioners (ECPs) is proposed.

This paper is based on a study on an Ambulance Service in the UK. There is a plan by the Ambulance Service to provide access to Electronic Patient Records (EPR) for the ECPs. The introduction of the EPR to the ECPs' work domain is expected to have positive impacts. A study was designed to evaluate SA and decision-making aspects of job performance. These cognitive variables are integrated within a framework to study how they are affected by the introduction of EPR.

\section{SITUATION AWARENESS AND HEALTHCARE}

Reviews of the literature on SA point to its 'various' and 'no universally accepted definitions' (for example, see Salmon et al., 2006). The variety of definitions reflects the different views and approaches in understanding SA. For example, some authors consider SA as a state of knowledge and an umbrella term for a range of cognitive processes (Rouseau, et al., 2004). SA is also viewed as a skill (McIlvaine, 2007). Endsley (1988, p 97) defined SA as “...the perception of the 


\section{Abd Hamid $\bullet$ Waterson $\bullet$ Hignett \\ Situation Awareness among Emergency Care Practitioner}

elements in the environment within a volume of time and space, the comprehension of their meaning, and the projection of their status in the near future".

Based on a review of SA definitions, Dominguez delineated four pieces of information that are necessary in a comprehensive definition of SA. That information relates to the processes of sensing, perceiving, and anticipating. The element of perceiving covers the processes of forming a mental model and its usage in cyclical perceptual process. Thus, SA is defined as "continuous extraction of environmental information, integration of this information with previous knowledge to form a coherent mental picture, and the use of that picture in directing further perception and anticipating future events" (Dominguez, 1994, p 11). This definition retains much of Endsley's definition. However, Dominguez's definition implies the use of a different theoretical framework (i.e. activity theory) than Endsley's model which explicitly shows individual (e.g. learning, memory stores, expectations) and system (interface design, stress, complexity) factors that affects the development and maintenance of SA.

Amidst these debates and criticisms, research progress on SA is being made. Among other reasons, the measurement of SA can be used to evaluate system design, evaluating training techniques, and understanding human cognition (Endsley and Robertson, 2000) including naturalistic decision-making (Endsley, 1997). Furthermore, SA is very useful for system evaluation (Tsang \& Vidulich, 2006), lending itself as a valuable tool for a systems ergonomics approach. This paper focuses on a subsystem of healthcare. Blanford and Wong (2004) had examined SA in emergency medical dispatch. This paper looks at ECPs within an ambulance service in England.

\section{EMERGENCY CARE PRACTITIONER (ECP)}

The ECP is defined as "a healthcare professional who works to a medical model, with the attitude, skills and knowledge base to deliver holistic care and treatment within the pre-hospital, primary and acute care settings with a broadly defined level of autonomy" (ECP Team, Skills for Health, 2007, p 9). In terms of what they do, an ECP is an "advanced practitioner capable of assessing, treating and discharging/referring patients at the scene" (Department of Health, 2005, p 51). Students who were trained to be ECPs said that the ECPs "occupies the space between the general practitioner, the nurse and the paramedic" (Doy \& Turner, 2004, p 365).

The development of the ECP role can be traced to at least two documents published by The Ambulance Service Association and Joint Royal Colleges Ambulance Liaison Committee (Modernisation Agency Department of Health, 2004) that recognise the need to create an autonomous role that can link different professions in emergency care (Cooper, et al., 2004). One drive for the creation of a new role is a year-to-year increase in 999 calls for urgent care (non-life threatening) while the demand for emergency care remains constant (The Ambulance Services Network, 2008). Thus, the ECP role was designed to reduce patient transport to the hospitals by catering to the demand for urgent care.

\section{Role Profile}

An ECP is "usually a paramedic or nurse who has undertaken specific training and education in order to be able to respond to the first contact needs of patients accessing urgent care" (Department of Health, 2005, pp 51). "The Competence and Curriculum Framework for the Emergency Care Practitioner" published by the Department of Health specifies the expected content of training programme for ECPs. According to this document, a training programme for ECPs should enable them "to make independent decisions based on sound clinical assessment and judgement, to complete episodes of care in a range of settings, including the out of hospital setting, when it is safe and appropriate, and to arrange appropriate referrals" (ECP Team, Skills for Health, 2007, p 9). The autonomy in making decisions is underscored by The Job Evaluation Handbook (DOH, 2003) that weighted the ECP's analytical and judgemental skills at 4 and responsibility for patient care at 5 (the maximum level).

\section{Performance Parameters and Evaluation of the Role}

An ambulance service control rooms receives 999 calls that would then be categorised into Category A, B, C, or urgent as listed in Table 1. The performance targets for each call category are different. For example, the benchmark is to meet the time target for at least $75 \%$ of the Cat A call (NHS Information Centre, 2008).

The performance target in terms of time is very clear. However, the vision on the quality of care is less so. A national evaluation of ECP schemes concludes that the "ECP schemes are moving forward in line with original objectives and could be having a significant impact on the emergency services workload" (Mason, et al., 2006, p 435). The dimensions covered in that study are patient outcomes, patient satisfaction, and cost. The estimated cost per EPC patient contact is 26, compared to 55 per Emergency Department (ED) contact. Another study also shows the cost saving in deploying ECP both in the urban and rural areas. The costs per response for an Emergency Ambulance are $£ 81$ (urban) and $£ 170$ (rural) while for the ECP the costs per response are $£ 58$ (urban) and $£ 108$ (rural) (Modernisation Agency Department of Health, 2004). 


\begin{tabular}{lll}
\hline Category of Calls & Types of Cases & Response time \\
\hline A & immediately life threatening & 8 minute response \\
\hline B & serious but not life threatening & 19 minute response \\
\hline C & not immediately serious or life threatening & locally agreed response time standards \\
\hline Urgent & request for transportation via a separate phone line & $\begin{array}{l}\text { usually in response to a GP/midwife/health } \\
\text { care professional }\end{array}$ \\
\hline
\end{tabular}

Table 1. Response time for different categories of calls.

Gray and Walker (2008) examined the reduction of admission to the ED. They compared cases where patients attended to by ECP to patients who went the ED. Patient data for two types of patients were studied: patients with breathing difficulties and elderly patients with a fall. The results show that the rates of admission to hospital are lower for patients attended to by an ECP both at initial contact and at 28 days. This study shows that ECP are effective at lowering the rates of admission to hospital. Similarly, Mason et al. (2007, p 239) found that "care provided by ECPs appears to reduce the need for subsequent referral to other emergency and unscheduled care services". Cooper et al. (2004) stated that there are advantages for extending the role of paramedics to that of ECPs with regards to deployment of resources, especially relating to non-conveyance, and improved their decision-making repertoire.

\section{A Decision-Making Framework for ECPs}

One decision that the ECP has to make for every patient is whether to treat the patients in situ or transport them to the hospital. There are other treatment pathways available - such as referral to a GP and social services - but for this analysis, the decision is simplified into transport or no transport. Additionally, the analysis uses signal detection theory as a metaphor to describe the different aspects of decision-making. The signal detection metaphor is similar to the model of recognition primed model described by Klein et al. (1993). As Figure 1 shows, the might be four possible outcomes of ECPs diagnosis based on the actual needs of the patients. In this analysis, two things are assumed: the outcomes depends on the incoming calls/jobs (i.e. the condition of the patients, the signal) and the diagnostics (detection) skills of the ECPs.

\begin{tabular}{llc|c|} 
& \multicolumn{2}{c}{ Actual Need } \\
& & Transport & Not transport \\
\cline { 3 - 4 } ECP's & Transport & Hit & False alarm \\
\cline { 3 - 4 } diagnosis & Not transport & Miss & Correct rejection \\
\cline { 3 - 4 } & &
\end{tabular}

Figure 1. Decision-making among ECPs.

If the control room pass randomly the calls from patients to the ECPs, it would be expected that there would be equal number of cases for both columns in Figure 1, giving a conveyance rate of 50\%. An ideal condition is one where the ECPs are assigned only calls that fit the right column (Actual Need - Not Transport). This condition would occur if ECPs are given only jobs appropriate for community treatment. This bias towards non-conveyance is in accordance with the rationale for creating the ECP role in the first place. Therefore, the possible error in this situation is a false alarm, where the ECP overestimated the need to hospitalise. False alarms are associated with increased financial cost as mentioned before. If the ECPs diagnosis is perfect, then all the cases would be located in the correct rejection region.

If the ECPs are assigned cases that necessitate transportation (left column in Figure 1), another type of error can occur. The ECP could miss patients who really need to be transported to the hospital. While there is financial saving made, the threat to patient safety and health outcomes increases. A bias in this direction could mean a decrease in the quality of healthcare provided to the patients.

The rate of conveyance can be examined to reveal biases in the system. Conveyance rate that deviate significantly from the ideal condition as described above indicates the presence of a bias. To identify this bias and understand the factors leading to the deviation, an analysis of the Ambulance Services system with specific focus on SA is proposed. Most of the studies on ECP examined post-task aggregated data. Studies that examine ECPs in action would complement the existing literature. Given what is stated, implied, and observed about ECP so far, there is value in investigating the dynamics of decision-making among ECPs. The objectives of the present study are to (1) map the communication of ECP within EMAS, and (2) develop a framework that links SA and decision-making among ECPs. 


\section{METHODS}

The scoping study adopted a participative observation approach. The observation was conducted during the participants' normal working hours and participants were nominated by the Ambulance Services. All of the participants are employed full-time. No compensation was given for their participation.

Ride-out sessions were organised with the aim of describing the nature of the role of ECP. The first author followed two ECPs during their regular shifts. The ECPs were shadowed two shifts each for a total of 36 hours. To increase the probability of attending a variety of patient cases, both day and night shifts as were covered during weekdays and weekends. The observation was structured around The System Engineering Initiative for Patient Safety (SEIPS) model of patient safety (Carayon et al. 2006). The SEIP model was chosen to aid the development of a domain-specific framework. Thus, during the observation, notes were made regarding machines and equipments, task procedure, related systems, environment, and task outcomes. In addition, SA related issues were noted based on Endsley's (1988) 3-level SA classification.

The researcher also participated in two sit-in sessions with three Triage Nurses at a control room of the Ambulance Services lasting four hours. Subsequently, the ECPs, and the triage nurse were interviewed. Based on the observation and interview data, a communication map of the ECP within the Ambulance Services was produced. This map was then validated by a subject matter expert (the Director of Operations for the Ambulance Service) and used to develop an integrated socio-technical system framework.

\section{FINDINGS AND DISCUSSION}

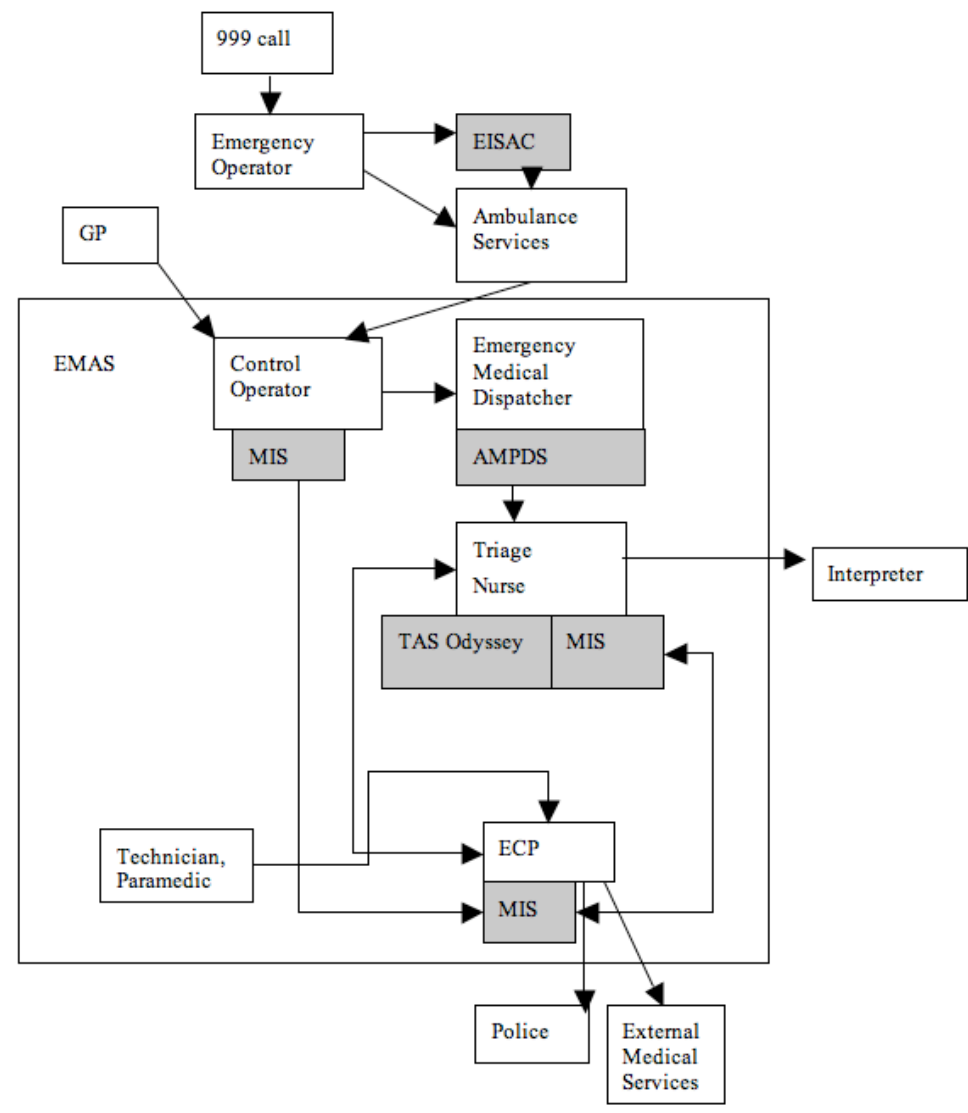

Figure 2. Communication map of the ECPs within EMAS.

As a system, the ambulance service is populated by different stakeholders and information systems. The ECPs operates physically inside this system (e.g. when being on call at the ambulance station) and also outside it (e.g. when treating patients at their home). More importantly the flow of communication is not easily defined by physical or geographical boundary. To further examine the communication flow, a communication map was produced.

Figure 2 is the communication map that was validated by a subject matter expert. It shows how a 999 call is filtered down in the control room and assigned to an ECP via the persons and information systems that they use. The TAS Odyssey (Telephone Clinical Assessment System) and AMPDS (Advanced Medical Priority Dispatch System) are used in triage work. Meanwhile, the MIS (Control CAS System ) allows the exchange of information between the different 
persons in the system. In this map, landline phones and mobile phones are excluded to highlight the information systems, rather than the devices. Based on this map, a socio-technical system framework was developed by taking into account the decision-making framework, and job design issues.

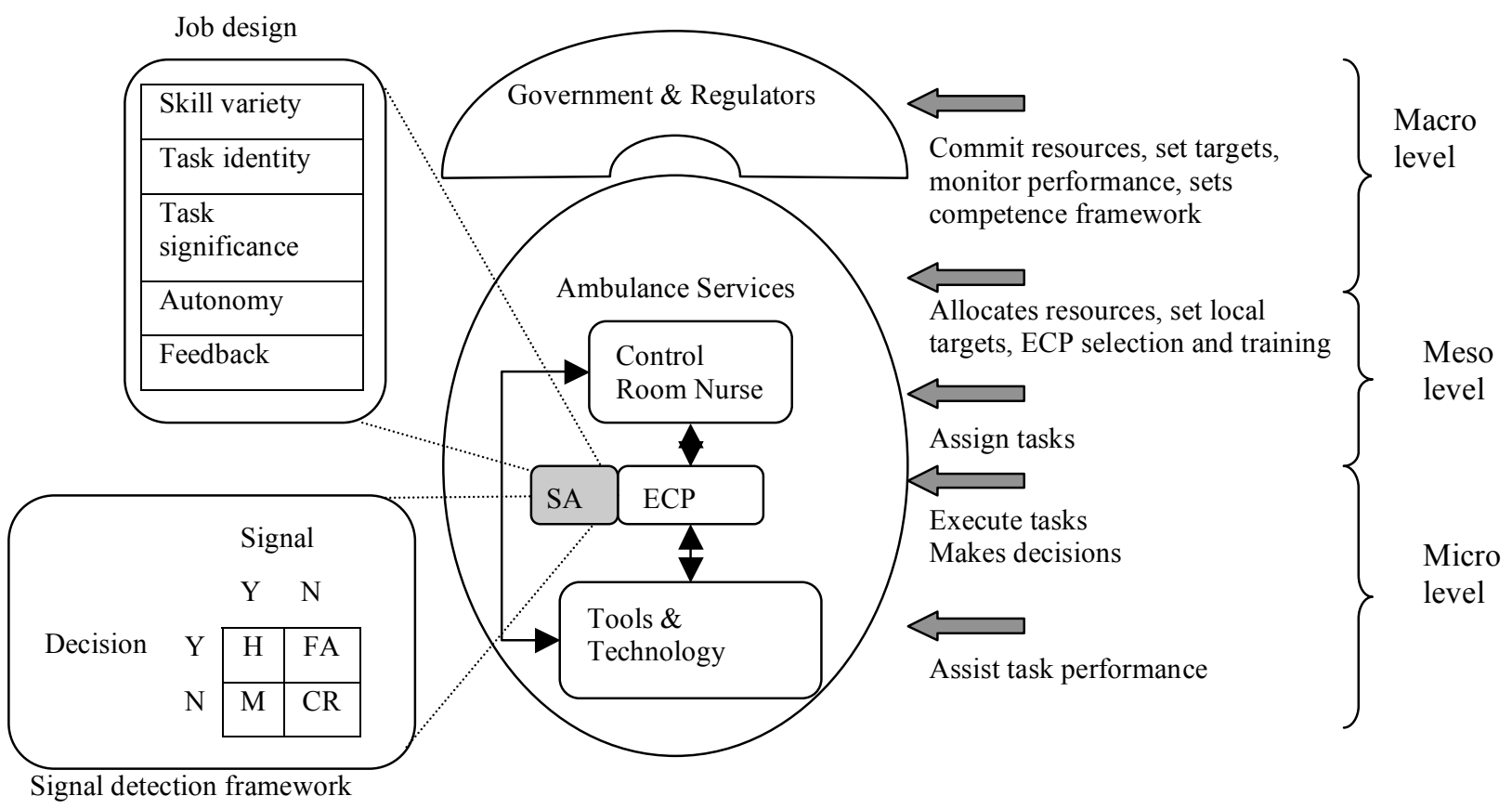

Figure 3. A socio-technical system framework for SA among ECPs.

Figure 3 is a static socio-technical system framework that incorporates signal detection and job design as attributes attached to the ECPs. The framework captures micro, meso, and macro level factors that may have cascading effect on SA and, consequently, decision-making.

For the purposes of illustration, consider the presence of noise in the ECPs' tasks. The noise in the system cascades down to the ECPs from the macro level (e.g. the management setting (or lack thereof) a policy regarding the role of ECPs - either perform a pure ECP jobs or a variety of jobs). The Control Room (meso level factor) can also add noises into the system. One ECP commented that the patient information from the control room (as displayed on the MIS screen) does not help much in assessing a patient's condition. He would have to speak to the Control Room staff directly to get information that is more accurate. The control room staff's SA does not seem to transfer well via the electronic communication technology. At the micro level, noise originating from the patient (e.g. pulse pattern) might affect SA as indicated in Table 2.

The thresholds for signal detection among ECPs are affected by experience and expectation. One ECP reported having more confidence in leaving the patients at home (equivalent to having a higher threshold) as a result of ECP training. In signal detection theory terms, the training affects the sensitivity by making it easier for the ECPs to detect the conditions in the patients that requires hospitalisation. Another ECP gave an anecdotal example of how a patient's renal disorder history was overlooked. This oversight may have been influenced by a stereotype (a form of expectation) associated with the patient's ethnic background.

The signal detection component of this framework underscores the uncertainties surrounding ECPs' decision-making. As described above, the uncertainties can be traced at all levels of the work system. Further use of the framework would be to identify ways to support SA and decision-making.

The ride out sessions revealed SA-related issues. Table 2 highlights issues at each of the three level of SA. For example, the threat to Level $1 \mathrm{SA}$ is due to the limited screen area of the MIS device installed in the ECP's vehicle. In the interview, the ECP reported that in the previous workplace (a different Ambulance Service), an incoming MIS message would automatically input the postcode into the satellite navigation system. The ECP thought that investment should be made with regards to the communication technology to enable him to meet the performance targets. The problem regarding the incomplete postcode can be compounded by relative unfamiliarity with local geography. The ECP who has just started working in the new area was not familiar with the street names. The information displayed on the MIS screen does not lead to an understanding (Level 2 SA) of the geographical destination. A rough estimate of the destination would have enable the ECP to respond faster by driving towards the general area, and fine-tuning the 
direction as more understanding develops. The personal factor can be mitigated by the management team by stationing and training the ECPs so that they have relative familiarity with their geographical catchment area. The example for level 3 SA in Table 2 shows how SA is related to a job design issue. The ECP reported that a feedback for a completed job would be beneficial. In other word, the feedback would constitute a learning component that is important for the development of accurate SA in the future.

\begin{tabular}{lll}
\hline SA Levels & Observation & Inference \\
\hline $\begin{array}{l}\text { Level 1: } \\
\text { Perception }\end{array}$ & $\begin{array}{l}\text { The MIS screen in the ECP's vehicle } \\
\text { displayed truncated postcode }\end{array}$ & $\begin{array}{l}\text { ECP cannot perceive complete } \\
\text { information }\end{array}$ \\
\hline $\begin{array}{l}\text { Level 2: } \\
\text { Comprehension }\end{array}$ & $\begin{array}{l}\text { ECP reported that ECG output is } \\
\text { different from what was expected based } \\
\text { on pulse pattern }\end{array}$ & $\begin{array}{l}\text { ECP cannot comprehend the } \\
\text { contradictory symptoms the patient } \\
\text { presented }\end{array}$ \\
\hline $\begin{array}{l}\text { Level 3: } \\
\text { Projection }\end{array}$ & $\begin{array}{l}\text { ECP reported that he wish he know } \\
\text { whether the patients are all right after } \\
\text { taken by the ambulance to the A\&E }\end{array}$ & $\begin{array}{l}\text { ECP cannot make accurate projection } \\
\text { of patients' future health condition }\end{array}$ \\
\hline
\end{tabular}

Table 2. Observations of SA issues in ECP work.

\section{FUTURE WORK AND CONCLUSIONS}

This paper described the development of a socio-technical system framework for SA and decision-making among ECPs. The proposed framework will be used to evaluate the introduction of Electronic Patient Record (EPR) in the ambulance service. EPR is seen as a potential tool to assist the ECPs making better decisions and actions. EPR refers to "the electronic collection of clinical narrative and diagnostic reports specific to an individual patient" (Safran \& Goldberg, 2000). It enables access to more information about the patient. This information can aid decisions on providing the most suitable treatment for the patient. The formulation of the decision can occur even when the ECPs are on their way to meet the patient. This time advantage is important considering the time criticality of some of their responses.

The benefits of EPR in terms of SA and decision-making had been documented in healthcare systems, albeit not in emergency medical services specifically. For example, Hertzum and Simonzen (2008) measured the effects of electronic records for three clinical activities: team conference, ward rounds, and nursing handover. Among the variables that showed a difference over paper-based documentation were mental workload, clarity about the importance of and responsibilities for work tasks, number of information missing, and quantity of messages to pass on, clarity of the status of the nursing plans for each patient. These findings are relevant to understanding SA among ECPs. The variables that were identified are micro level variables related to SA. In another study, Hippisley-Cox et al. (2003) also compared EPR with paper-based record. Their findings also point to the potential of EPR to support a better SA. For example, the EPR improves Level $1 \mathrm{SA}$ (more paperless records were fully understandable and fully legible).

However, Ludwick and Ducette's (2009) review of EPR literature shows that quality of care, patient safety and provider/patient relations were not, positively or negatively, affected by systems implementation. In the review, they examined factors affecting implementations of EPR. Among the factors identified are systems' graphical user interface (micro level factor), and project management (meso level factor) and procurement (macro level factor). Their review of EPR literature also revealed the concept of socio-technical factors, or "fit" factors, that complicate health information systems deployment. These studies point to system factors that could affect SA negatively.

The studies cited above show the potentials and advantages of the EPR. Whether these potentials and advantages are generalisable to the ECPs in Ambulance Services is subject to further studies. A study on the ECPs would help in determining the actual content that needs to be designed into the EPR for their use. Referring back to Table 1, EPR are expected to yield benefits in term of support for SA and decision-making. Several relevant questions at this juncture are:

1) How different is the SA of ECPs before and after arriving at scene to treat patients?

2) Does EPR affect the signal detection pattern of ECPs?

3) Which job design issues is most related to SA?

4) Does EPR add to medical services' resilience through supporting SA and decision-making? 
In conclusion, future work will be carried out to evaluate the introduction of EPR within the proposed socio-technical system framework. This effort would allow further examination of SA in the healthcare systems by linking it with decision-making.

\section{ACKNOWLEDGMENTS}

We thank the Ministry of Higher Education Malaysia, and the International Islamic University Malaysia for financial support to the first author, and to Dave Whiting of NHS, UK for his support to this project.

\section{REFERENCES}

Blanford, A., \& Wong, W. (2004) Situation awareness in emergency medical dispatch. International Journal of Human-Computer Studies, 61(4), 421-452.

Carayon, P., Hundt, A.S., Karsh, B.T., Gurses, A.P., Alvarado, C.J., Smith, M., Brennan, P.F. (2006), Work system design for patient safety: The SEIPS model. Quality and Safety in Health Care, 15 (Suppl. I), 50-58.

Cooper, S., Barrett, B., Black, S., Evans, C., Real, C., Williams, S., \& Wright, B. (2004) The emerging role of the emergency care practitioner. Emergency Medicine Journal, 21, 614-618.

DOH. (2005) Taking healthcare to the patient. Transforming NHS Ambulance Services. London: DOH Publications.

Department of Health. (2003) Job evaluation handbook. London: DOH Publications.

Dominguez, C. (1994) Can SA be defined? In M. Vidulich, C. Dominguez, E. Vogel, \& G. McMillan. Situation Awareness: Papers and annotated bibliography (U). Ohio: Armstrong Laboratory, Human System Center.

Doy, R., \& Turner, K. (2004) The giraffe: the emergency care practitioner; Fit for purpose? The East Anglian experience. Emergency Medicine Journal, 21, 365-366.

ECP Team. Skills for Health. (2007) The competence and curriculum framework for the emergency care practitioner. Bristol, Department of Health.

Endsley, M.R. (1988) Design and evaluation for situation awareness enhancement. Proceedings of the Human Factors Society 32nd Annual Meeting. Santa Monica, CA: Human Factors Society. pp 97-101.

Endsley, M.R. (1997) The role of situation awareness in naturalistic decision-making. In C. E. Zsambok, \& G. Klein, (Eds.) 1997. Naturalistic decision-making, Erlbaum, Hillsdale, NJ, pp. 269-284.

Endsley, M.R., \& Robertson, M.M. (2000) Training for situation awareness in individuals and teams. In M.R. Endsley, \& D.J. Garland (Eds.) Situation Awareness Analysis and Measurement. London: Erlbaum. pp 349 - 365.

Gray, J.T., \& Walker, A. (2008) Avoiding admissions from the ambulance service: a review of elderly patients with falls and patients with breathing difficulties seen by emergency care practitioners in South Yorkshire. Emergency Medical Journal, 25, 168-171.

Hertzum, M., \& Simonsen, J. (2008) Positive effects of electronic patient records on three clinical activities. International Journal of Medical Informatics.

Hippisley-Cox, J., Pringle, M., Cater, R., Wynn, A., Hammersley, V., Coupland, C., Hapgood, R., Horsfield, P., Teasdale, S., \& Johnson, C. (2003) The electronic patient record in primary care-regression or progression? A cross sectional study. BMJ, 326, $1439-1443$.

Klein, G.A., Orasanu, J., Calderwood, R., Zsambok, C.E. (Eds.). (1993) Decision making in action: Models and methods. Norwood, NJ: Ablex Publishing Corporation.

Ludwick, D.A., \& Doucette, J. (2009) Adopting electronic medical records in primary care: Lessons learned from health information systems implementation experience in seven countries. International Journal of Medical Informatics.

Mason, S., Coleman, P., O’Keefe, C., Ratclife, J., \& Nicholl, J. (2006) The evolution of the emergency care practitioner role in England: Experiences and impact. Emergency Medical Journal, 23, 435 - 439.

Mason, S., O’Keeffe, C., Coleman, P., Edlin, R., \& Nicholl, J. (2007) Effectiveness of emergency care practitioners working within existing emergency service models of care. Emergency Medical Journal, 24, 239-243.

McIlvaine, W.B. (2007) Situational awareness in the operating room: a primer for the anaesthesiologist. Seminars in Anesthesia, Perioperative Medicine and Pain, 26, 167-172.

Modernisation Agency Department of Health. (2004) The Emergency Care Practitioner Report. London: COI Communications for the Department of Health. 
NHS Information Centre (2008) Ambulance Services, England, 2007-08. London: The Information Centre.

Safran, C., \& Goldberg, H. (2000) Electronic patient records and the impact of the Internet. International Journal of Medical Informatics, 60, 77-83.

The Ambulance Service Network. (2008) A Vision For Emergency and Urgent Care: The Role of Ambulance Services. London: The NHS Confederation.

Tsang, P.S., \& Vidulich, M.A. (2006) Mental workload and situation awareness. In G. Salvendy (Ed). Handbook of Human Factors and Ergonomics. $3^{\text {rd }}$ ed. Hoboken, NJ: John Wiley \& Sons, pp $243-268$.

Rouseau, R., Trémbley, S., \& Breton, R. (2004) Defining and modelling situation awareness: a critical review. In S. Banbury, \& S. Tremblay (Eds.) A Cognitive Approach to Situation Awareness: Theory and Application. Aldershot, Hampshire, UK: Ashgate Publishing. pp $3-21$. 